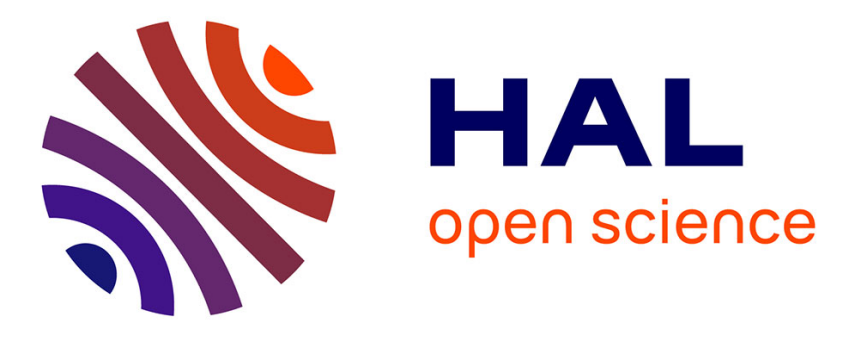

\title{
Mixed-integer linear programming for multibeam satellite systems design: Application to the beam layout optimization
}

Jean-Thomas Camino, Christian Artigues, Laurent Houssin, Stéphane

Mourgues

\section{To cite this version:}

Jean-Thomas Camino, Christian Artigues, Laurent Houssin, Stéphane Mourgues. Mixed-integer linear programming for multibeam satellite systems design: Application to the beam layout optimization. 2016 Annual IEEE Systems Conference (SysCon), Apr 2016, Orlando, FL, United States. pp.1-6, 10.1109/SYSCON.2016.7490613 . hal-01351492

\section{HAL Id: hal-01351492 \\ https://hal.science/hal-01351492}

Submitted on 4 Aug 2016

HAL is a multi-disciplinary open access archive for the deposit and dissemination of scientific research documents, whether they are published or not. The documents may come from teaching and research institutions in France or abroad, or from public or private research centers.
L'archive ouverte pluridisciplinaire HAL, est destinée au dépôt et à la diffusion de documents scientifiques de niveau recherche, publiés ou non, émanant des établissements d'enseignement et de recherche français ou étrangers, des laboratoires publics ou privés. 


\title{
Mixed-Integer Linear Programming for Multibeam Satellite Systems Design: Application to the Beam Layout Optimization
}

\author{
Jean-Thomas Camino $^{(1)(2)}$, Christian Artigues ${ }^{(2)}$, Laurent Houssin ${ }^{(2)}$, Stéphane Mourgues ${ }^{(1)}$ \\ ${ }^{(1)}$ Airbus Defence and Space, Space Systems, Telecommunication Systems Department, \\ 31 Rue des Cosmonautes, 31402 Toulouse, France \\ (2) LAAS-CNRS, Université de Toulouse, CNRS, UPS, Toulouse, France
}

\begin{abstract}
In a society where the demand for multimedia applications and data exchange is experiencing an unstoppable growth, multibeam systems have proven to be one of the most relevant solutions for satellite-based communication systems. Though already well represented among the geostationary satellites today, there are still several unresolved design optimization challenges for these complex systems that could lead to improved performances and to better system costs. The satellite platform, the repeater, and the antennas are examples of subsystems that should be designed jointly in order to reach an optimized technical solution that fulfills the service requirements. Traditionally, such complex tasks are addressed through a decomposition of the overall system design into a sequence of smaller decision problems. In this article, we propose to rely on operations research techniques to, on the one hand, take into account explicitly the interdependencies of these decomposed problems, and on the other hand, to handle the own constraints of each subsystem and their interactions. In this paper, the focus is laid on the optimization of the beam layouts of the multibeam satellites. Indeed, in addition to being a perfect example of the aforementioned importance of dealing with subsystem constraints, this problem appears early in the chain of design of a multibeam satellite system and is therefore critical for the quality of the telecommunication system: the weaknesses of a beam layout cannot be made up for later on in the system design. For this crucial optimization phase, the strength of the methodology we propose in this paper is to use mixed-integer linear programming to incorporate explicitly technological feasibility constraints of the subsystems involved, while preparing at best the subsequent design problems. Most importantly, our approach allows to overcome several resisting flaws of the already existing algorithms.
\end{abstract}

Keywords: Satellite Telecommunications, Systems Design, Mixed-Integer Linear Programming, Operations Research

\section{INTRODUCTION}

On the service area of a given satellite system, any user can be reached regardless of its location. This is particularly interesting for rural areas for which deploying a terrestrial network is hard or even impossible. This unique characteristic of the satellite systems combined with their ability to offer all kinds of telecommunication services (television, radio, telephone, internet...) have made them indispensable in our ultra-connected society. A multibeam satellite is a particular type of telecommunication satellite characterized by a plurality of relatively narrow beams used to provide coverage to its service area. As discussed further in the article, compared to systems that use only one large beam to cover the entire service area,

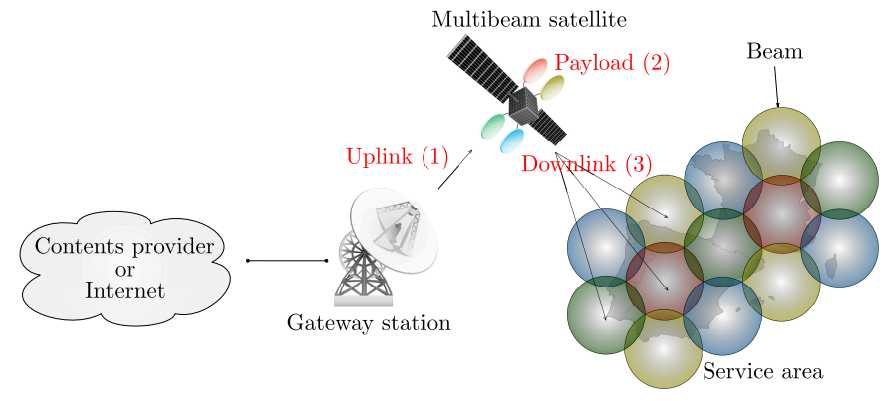

Fig. 1. Forward link of a multibeam satellite system

the antenna gains are significantly improved with the multibeam solution. Plus, since two beams that are sufficiently separated spatially are allowed to reuse the same frequency, opting for a multibeam coverage is a way to make the most of the available system bandwidth which is a really expensive resource in any telecommunications system. As shown in Fig. 1, one or several gateways (ground stations) are connected through the terrestrial network to producers of multimedia content or to the internet backbone, depending on the service provided by the satellite. Radio frequency signals are generated from these gateways in direction of the satellite in the uplink, denoted by (1) in Fig. 1. The role of the satellite payload (2) (composed of "receive" and "transmit" radiofrequency antennas and of the so-called "repeater") is then to receive, downconvert in frequency, amplify in power, and retransmit these signals in the different beams of the downlink (3) where the end-users are located and equipped with terminals allowing them to receive the content delivered by the satellite. This is called a "forward link", which in some cases (internet service by satellite for instance) is completed by a "return link" from the users to the gateways. This standard system architecture of Fig. 1 is the one we chose to consider in this study.

Reaching the high data rates aimed in the telecommunications services today raises several system level challenges for multibeam satellites, and has therefore motivated a significant amount of research. In [13], the state-of-the-art of these systems is summarized and promising techniques to improve the system performances are analyzed: wider spectrum allocation, frequency reuse schemes, feeder link design (between the gateways and the satellite) and interference mitigation techniques. In [10], the authors highlight the interest of relying on optical communications instead of traditional 
radiofrequency signals for the feeder link of geostationary satellite systems, and propose a methodology and algorithms to tackle the several design issues that these innovative system architectures raise. The authors of [5] analyse multibeam systems that use two co-localized multibeam satellites that are coordinated to provide enhanced and more robust performances. Some research papers focus exclusively on one of the satellite subsystems: for instance, the authors of [12] investigate algorithms to optimize the design of multibeam array antennas. For a given system architecture and a given satellite payload, literature is also rich on the question of how to optimize the allocation of resources such as the satellite power and the system bandwidth: [9], [6], [7], [14] and [11] for instance. On the other hand, the stress is rarely put on the strong interdependencies of all the subsystems of a multibeam satellite system in the design phase. Under the assumption that the system architecture has already been chosen and is the one of Fig. 1, the objective of this article is indeed to demonstrate that, when some crucial design problems are not solved jointly for the different subsystems involved, then, whatever the quality of the following allocation of resources and individual subsystem optimization processes, the overall system solution will be either technologically infeasible or perfectible in terms of cost or performances. We also show that in the case of the complex systems that are the multibeam satellite networks, the process of making optimized technical decisions can be greatly enhanced if one relies on operations research techniques to handle the numerous constraints and criteria of all the different subsystems that interact. In this context, the beam layout optimization problem is presented and an example of algorithmic solution is provided and compared to the literature on the subject.

The rest of the article is structured as follows. Section II defines precisely the telecommunication mission requirements that are at the start of any multibeam system. They are derived into a set of design challenges, the stress being put on the space segment optimization for which a design process is proposed. In section III, the crucial beam layout optimization step is tackled with operations research techniques and more particularly mixed-integer linear programming: the assumptions made and the mathematical models are detailed. Section IV presents experimental results and comparisons with other algorithmic solutions. The conclusion and the way forward are discussed in section $\mathrm{V}$.

\section{TELECOMMUNICATION MISSIONS AND CORRESPONDING DESIGN CHALLENGES}

The requirements of the telecommunication missions can be of different types. In their most general form, they consist of a series of specific services that should be provided to designated regions of the Earth: television, radio, telephone, broadband applications (internet, videoconferencing...) to name a few. Both the quality and the quantity of these services can be specified. In addition, there might be for instance cost constraints on the overall system. Here, we will consider requirements inspired by broadband applications, where each point defined on the service area has its own traffic request expressed in Megabits per second, as illustrated with the fictitious scenario of Fig. 2 (generated randomly). From this specification of needs, the satellite system manufacturers must propose a whole solution including details on the ground segment (the gateways and the control centers), the user segment (satellite terminals), and the space segment (one multibeam satellite in the case considered here). Each of these three complex systems can be split in several subsystems with their own particularities and design challenges. It is impossible today, either numerically or manually by experienced engineers, to design globally the three segments of a multibeam satellite system: there are too many decisions to make on numerous interacting subsystems, and computationally heavy system simulations to assess the performances of the selected designs. The standard way to deal with such an issue

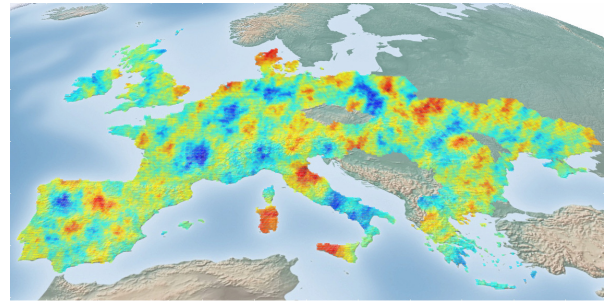

Fig. 2. Example of traffic demand map

is to divide the problem into simpler design questions that are chosen as to have a series of subproblems to solve that are as independent as possible. This very principle naturally leads to a division of the system design into the separate designs of the ground, user, and space segments. In this article, we assume that the ground segment and the user segment have already been designed: the number of gateways, their power, their position, the aggregate available system bandwidth, and the types of user terminals are known. As a result, it is possible to focus exclusively on the space segment and to discuss design approaches that can handle the various constraints (technological feasibility) and criteria (complexity, cost, weight) of the satellite payload, aswell as those dictated by the system requirements (see Fig. 3). Ideally, they should be handled all at once, but this global optimization would also be too complex to be effective in practice. Therefore, it is once again necessary to cleverly decompose the

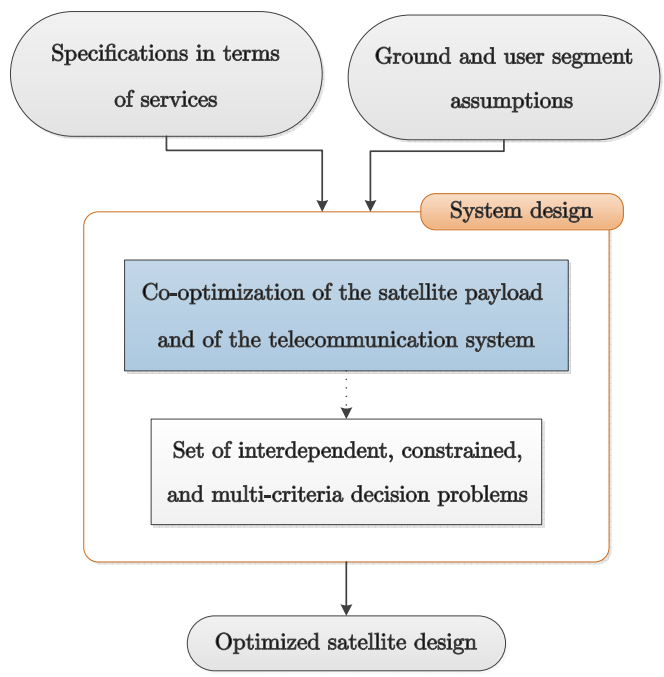

Fig. 3. Overview of the space segment design

system design. In the following section, such a decomposed process is proposed with the stress being put on one crucial resulting design problem: the beam layout optimization.

\section{BEAM LAYOUT OPTIMIZATION THROUGH MIXED-INTEGER LINEAR PROGRAMMING}

In Fig. 1, we have an example of multibeam coverage with 13 beams over European countries. Note that, for a radiofrequency antenna, the antenna gain is a measure of the power concentration of the radiated electromagnetic signals, value therefore defined for each direction in three dimensions. Each beam is the representation of a certain antenna gain loss $(-3 \mathrm{~dB}$ is a commonly encountered threshold for instance) relatively to the peak gain value for the corresponding satellite radio source: as a first approximation, the rule is that the gain decreases when the angular distance between the considered direction 
and the peak direction increases. Also note that the bigger the beam, the less the peak antenna gain for this beam, which explains why multibeam systems are attractive compared to single beam systems that cover the same regions with only one large beam. From the point of view of the satellite, and looking in direction of the Earth, common satellite antenna designs lead to beams that can be represented by disks on the surface of the Earth. This results in the definition of the "beamwidth" which is simply the diameter of such disks. We will say that a station is covered by a beam if its disk representation contains this station. For a given service area, defining a beam layout means deciding what will be the number of beams, the position of the beam centers on the surface of the Earth, the beam diameters, the mapping of the user stations to the different beams, and the satellite reflector antenna associated to each beam. Note that the performances and cost of a broadband satellite system are expected to differ notably from one beam layout to another according to how the heterogeneity of the traffic demand (case considered) over the regions to cover is handled. One key remark is that the common beam layout solutions are not well suited for heterogeneous traffic demands (see III-B). On the other hand, potentially more appropriate solutions can cause a significant increase on the payload complexity or can compromise the technological feasibility, which needs to be controlled from the start when the beam layout is defined. This is why we decided to devote particular attention to this already very complex optimization: the beam layout is the first step of the space segment design we propose in Fig. 4. As discussed earlier, the only way this process can be effective is by making explicit the links between the different design steps. To that end, we chose to propose an algorithmic solution that defines the beam layout under antenna and repeater constraints to create favorable conditions for the subsequent design optimization phases: antenna and repeater design. Of course, the required system performances and cost are already targeted during this first optimization. These constraints and objectives are detailed in the following section.

\section{A. Considered constraints and objectives}

\section{1) Platform constraints :}

The main platform constraint we will consider is the limit on the number of beams that can be embarked on the spacecraft. This can be due to either power, cost, mass or accommodation limitations. In any case, we will explicitly integrate this upper-bound on the number of beams in our beam layout optimization algorithm.

\section{2) Antenna constraints :}

As illustrated in Fig. 1, each beam is transmitted by exactly one satellite reflector antenna (4 of them are represented, each one characterized by its own color). Under the assumption adopted throughout this study that Single Feed Per Beam antennas are used (one radio source per beam), the main technological constraint is that, for each layout considered, there must exist a mapping of the beams to the different reflector antennas for which there is no overlapping in the corresponding blocks of sources. This leads to a minimum angular distance from the satellite point of view between two beams associated to the same reflector antenna, that distance being a function of the size of the sources. This technological constraint is represented in Fig. 5 where some elements of the antenna geometry are represented: the focus $O$, the focal length $F$, the slant focal length $F_{s}$, the diameter $D$ (projection of the parabola diameter on the focal plane), and the angular distance between the beams $\Delta_{\theta}$ caused by the inter-source distance $\Delta_{i, j}$. The important property is that using larger beamwidths requires larger sources to concentrate the illumination on a reduced reflector surface, which leads to greater minimum inter-sources distances and, therefore, to greater minimum separation distances. In practice, the rule we decided to use for the remainder of the study was to assume that the minimum angular separation for two beams transmitted by the same reflector is directly proportional to the mean of their two beamwidths, according to a coefficient $\kappa \in \mathbb{R}^{+}$such that

$$
\frac{3}{2} \leq \kappa \leq \sqrt{3}
$$

This range is representative of commonly encountered configurations for such Single Feed Per Beam satellite systems.

\section{3) Repeater limitations constraints :}

Note that the traffic demanding stations are assigned to the beams leading to an aggregate traffic request per beam. While these demands are a result of the beam layout optimization procedure, they are strongly connected to the following payload design phases. For instance, we can define for each beam a maximum traffic demand (that would depend on the beam diameter) above which we lose the guarantee that there will exist a feasible repeater architecture (hardware, frequency plan and bandwidth allocation) that can answer properly to that demand. This is exactly the constraint we implemented to make sure that the following payload design challenges can be resolved.

\section{4) Objective :}

Each station is characterized by its own traffic demand. The objective we decide to target is the maximization of the aggregate covered traffic, the ideal goal being $100 \%$. Note that the traffic of a given station is considered covered when this station is covered by at least one beam and assigned to one of them.

\section{B. Example of solutions to the beam layout problem}

In this section, a concrete scenario is analyzed to understand why there is a need for non-uniform beam layouts. In Fig. 6, the same traffic demanding service area has been covered by three distinct beam layouts. The first two are regular layouts with respectively 117 beams of $0.30^{\circ}$, and 50 beams of $0.5^{\circ}$. The third layout is a nonuniform one with 48 beams that combine the two beamwidths. We assumed that 4 antenna reflectors were available on the spacecraft and that $\kappa=\sqrt{3}$ for the angular separation constraint of equation (1). For the feasibility of the frequency plan and of the repeater, a maximum traffic per beam has been defined per beamwidth (higher threshold in the case of the smaller beams). In all three cases, more than $99.5 \%$ of the traffic is covered, so as far as what concerns the objective, there is no way to differentiate the three solutions. On the left (subfigures (a), (c) and (e) of Fig. 6), the mapping of the beams to the satellite reflectors is represented (one color per reflector) to examine the angular separation constraint. We observe that in the case of the regular layouts, the antenna constraint is fully satisfied: the mapping of the beams is natural and follows a repeated pattern. On the other hand, for the irregular beam layout, this assignment of reflectors is a real challenge and we can observe that some couples of large beams using the same reflector are closer than in the case of the regular layouts: this solution is not fully compliant with the antenna constraints. Yet, if we had managed to deal properly with the full set of antenna constraints, the irregular layout would have been way more interesting. Indeed, it uses less beams that the two regulars solutions, but most importantly, the balancing of the traffic is more appropriate and allows to satisfy all the maximum load per beam constraints. On that regard, the subfigures (b), (d) and (f) of Fig. 6 provide the load of each beam for each layout (blue is for the low values and red for the high values). The first regular layout with 117 small beams uses too many beams and most of them have really low demands (which is not cost-effective for the hardware embarked), and one of them exceeds its maximum value allowed (the most red beam). With the regular layout of 50 beams, this time the number of beams is acceptable and the traffic is well balanced in average, but several beams exceed their maximum load limits, leading to impossibilities to find a bandwidth allocation and a corresponding payload architecture that could satisfy these demands. In the irregular 


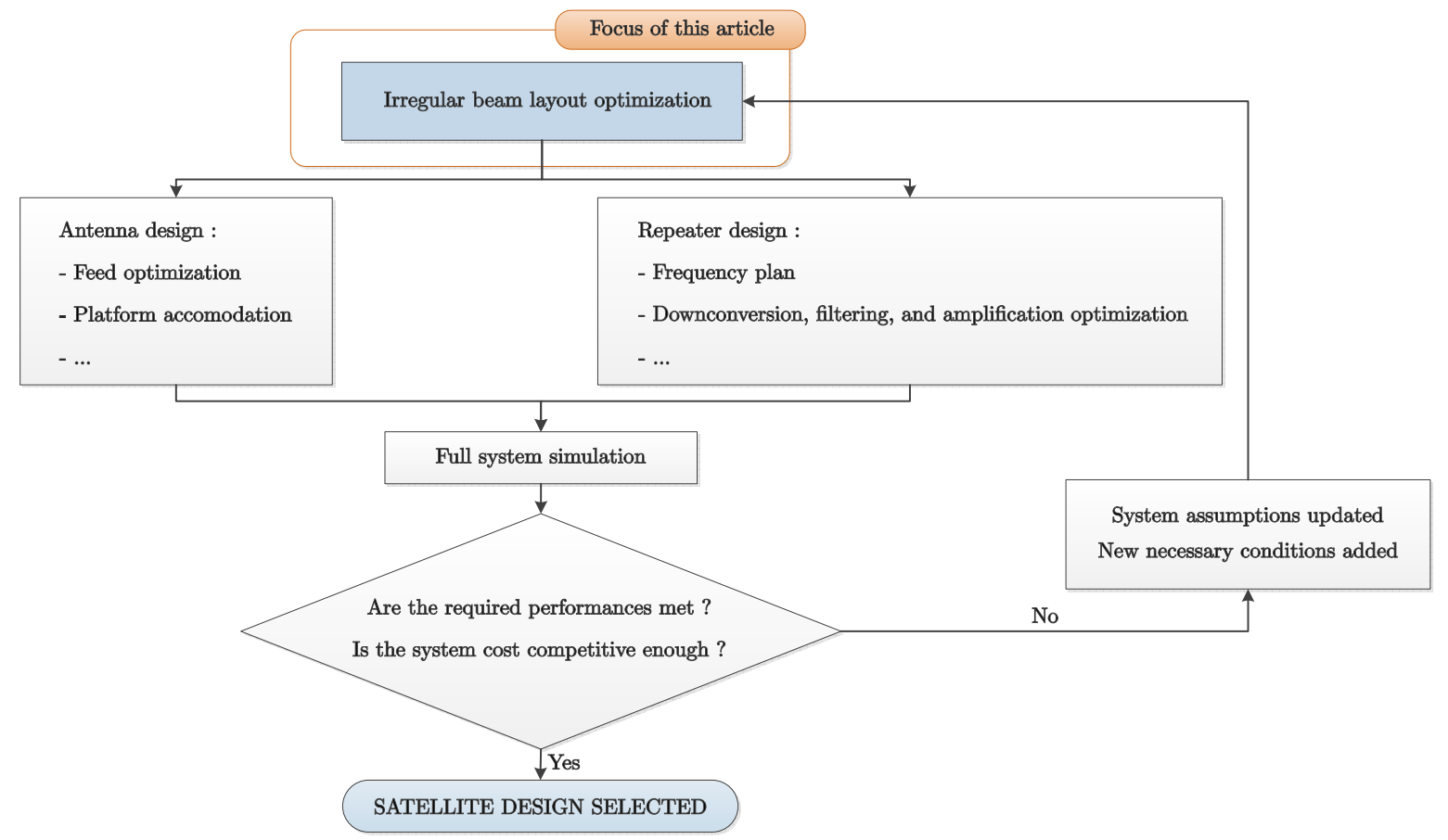

Fig. 4. Decomposition of the space segment design and corresponding process

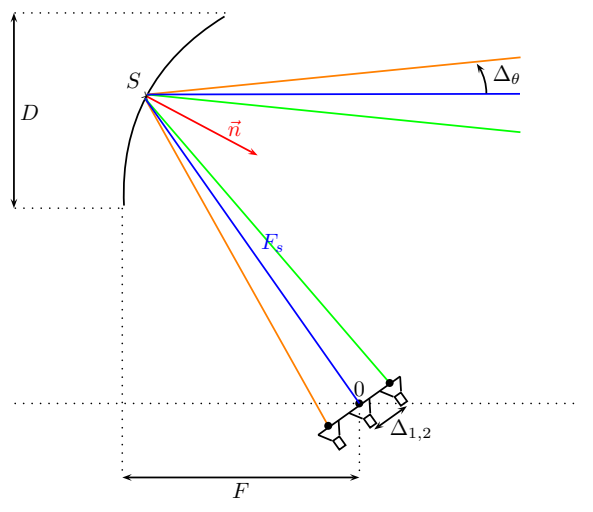

Fig. 5. Single Feed Per Beam antenna separation constraint

layout, the traffic is well distributed among the different beams and none of them exceed the traffic demand upper-bound: this is exactly what we will be looking for with our algorithm, with full antenna compatibility.

\section{Existing work}

The previous section showed that the common technique that uses a single beamwidth and the hexagonal lattice to cover the entire region is not appropriate for heterogeneous traffic demands. Yet, this pattern is known to maximize the density of circle packing (see [4] for instance), and would be optimal for a traffic demand perfectly homogeneous in space and in intensity. Also, the antenna feasibility (see III-A2) has been proven for these regular layouts for a spacecraft equipped with 3 or 4 reflectors which is a strong enough reason to continue to rely on them regularly. On the topic of breaking this regularity of the layouts, some litterature can be found. The authors of [1] suggest to aim at a load-balanced distribution of these demands within the different beams through an adjustment of their widths,

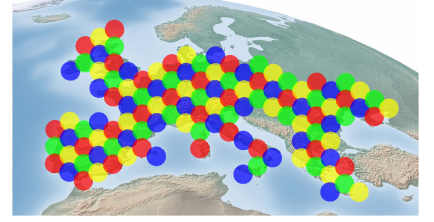

(a)

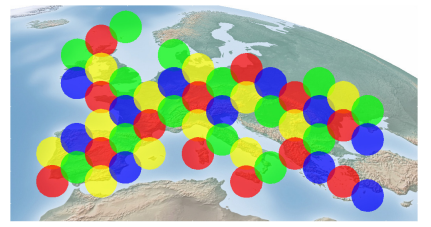

(c)

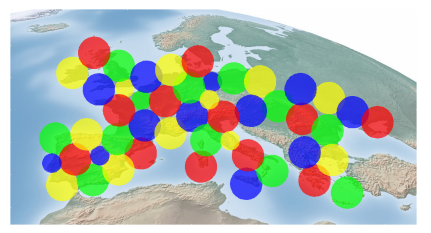

(e)

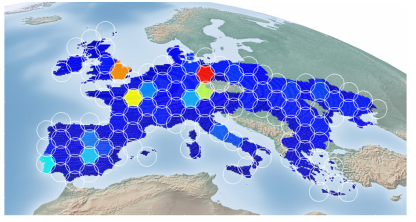

(b)

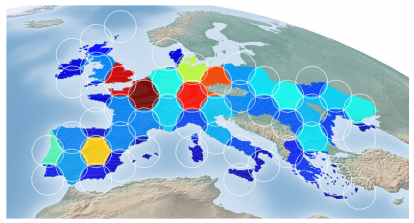

(d)

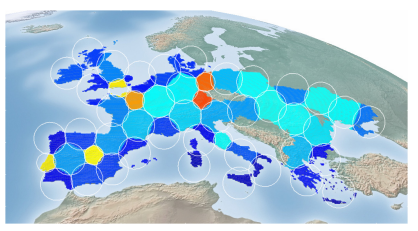

(f)
Fig. 6. (a),(c),(e) Satellite reflector mapping: 117 regular beams, 50 regular beams, 48 irregular beams (b),(d),(f) Load per beam: 117 regular beams, 50 regular beams, 48 irregular beams (blue $=$ low values, red $=$ high values)

the main idea being that imbalances of traffic demands inside the beams can lead to user stations being in the end either overloaded or underloaded in terms of offered capacity. The method proposed uses a partitionning of the region into evenly loaded polygonal subparts, each one of these being then treated individually to determine what beamwidth should be used in it and where the beam should be placed through an analysis of its shape and surface, thus defining the full 
layout. In that work, the question of the mapping of the beams to the antenna reflectors is not addressed. Furthermore, in [8] the authors observed that a drawback of this last method is that several zones remain uncovered or poorly covered. To overcome this issue, they use artificial neural networks and more precisely self-organizing maps that treat all the traffic demands in order to define sub-areas, each sub-area corresponding to one of the available beamwidths. The reasoning behind this method is that using narrow beams on the densest zone is a way to increase the antenna gains and the frequency reuse where it is the most needed, while using larger beams in the least dense areas can be sufficient to reach compliance. Beams are then placed in each sub-area in such a way as to minimize the uncovered areas, the regular lattice being used locally. Once the beams are placed over the service zone, the authors tackle the issue of the antenna configuration with a reflector allocation to the beams that includes a maximization of the angular distances between the beams coming from the same antenna, that NP-hard problem being solved heuristically. But the major drawback of treating the placement of the beams and their allocation to the antenna reflectors sequentially is that one is very likely to discover that, with a layout built regardless of the antenna constraint, even the optimal mapping of the beams to the reflectors can turn out to be technologically infeasible for the Single Feed Per Beam antennas. Guided by a combinatorial model of the problem, [3] proposes a heuristic procedure combining a randomized multi-start greedy approach and graph coloring to find jointly interesting positions for the beams and an acceptable beam mapping to the reflectors, with an upper-bounded number of beams and a pre-processing of the beamwidths to use.

\section{Mixed-integer linear programming model}

We propose in Fig. 4 a space segment design process in which sizing problems of acceptable complexity emerge. Reducing the design to a series of simpler problems is numerically interesting but is risky in the sense that it is necessary to make sure that the subsequent design problems in the process flow will be feasible, and in the best conditions given all the decisions already made. To that end, we decided to rely on operations research and more particularly on mixed-integer linear programming to solve the beam layout optimization problem with the constraints and objective of section III-A. The strength of the model we propose, besides being able to handle the subsystems interactions, is to handle actively the positioning of the beams: for each beam, there are variables defining its center. Most importantly, as opposed to what we can find in the litterature on the subject, we propose a model that allows to keep the domain of centers continuous. To do so, we observed that the beam positioning can be entirely done in the Euclidean plane in the right coordinate system (the true view angles coordinate system, see [2]), and we rely on results on the inner product in the plane to handle the angular distances through a discretization of the directions $[0,2 \pi[$. The mathematical model is presented in Fig. 7 , voluntarily without too many details on how we reached it. The goal is really to show that mathematical expressions can capture the interconnections of the satellite subsystems and, therefore, that operations research can be a good tool for the system engineer facing complex design problems. In the model of Fig. $7, \mathcal{B}=\left\{1, \cdots, N_{B}\right\}$ is the set of beams that can be placed on the service area, $N_{B}$ being the maximum number of beams allowed by the satellite platform. $\mathcal{S}=\left\{1, \cdots, N_{S}\right\}$ denotes the set of user ground stations, each station $s \in \mathcal{S}$ being characterized by its traffic demand $T_{s} \in \mathbb{R}^{+}$and its coordinates $\left(X_{\text {stations }, s}, Y_{\text {stations }, s}\right)^{T} \in \mathbb{R}^{2} . \mathcal{U}=\left\{1, \cdots, n_{\text {directions }}\right\}$ is the set of discretized directions of the Euclidean plane, and for each $u \in \mathcal{U},\left(U_{u, x}, U_{u, y}\right)^{T} \in \mathbb{R}^{2}$ are the coordinates of a unit vector characterizing that direction. $\mathcal{R}=\left\{1, \cdots, N_{R}\right\}$ is the set indexing the $N_{R}$ reflector antennas, and $\mathcal{W}=\left\{1, \cdots, N_{W}\right\}$ is the (finite) set of possible beamwidths: $W_{1}, \cdots, W_{N_{W}} \in \mathbb{R}^{+}$. For each beamwidth $w \in \mathcal{W}$, we define an upper-bound for the traffic assigned to a given beam: $\Gamma_{w} \in \mathbb{R}^{+} . \alpha_{s, b}, \omega_{b, w}, \rho_{b, r}, a_{b} \in\{0,1\}$ are binary variables that represent respectively: the covering (" 1 ") or not (" 0 ") of station $s$ by beam $b$, the choice (" 1 ") or not ("0") of beamwidth $w$ by beam $b$, the choice ("1") or not (" 0 ") of reflector $r$ by beam $b$, and the fact that beam $b$ is active (" 1 ") or not (" 0 "). There are further binary variables $\left(\beta_{b, b^{\prime}}, \gamma_{b, b^{\prime}, u}\right)$ and real positive coefficients $\left(M_{s}, N_{s}, N\right)$ that are only here to express correctly, and relax when needed, all of our system constraints.

$$
\text { Maximize } \sum_{(s, b) \in \mathcal{S} \times \mathcal{B}} T_{s} \alpha_{s, b}
$$

under the following constraints

$$
\forall s \in \mathcal{S}, \quad \sum_{b \in \mathcal{B}} \alpha_{s, b} \leq 1
$$

$\forall s \in \mathcal{S}, \forall b \in \mathcal{B}, \forall u \in \mathcal{U}$,

$$
\begin{gathered}
\left(\begin{array}{c}
x_{b}-X_{\text {stations }, s} \\
y_{b}-Y_{\text {stations }, s}
\end{array}\right)^{T}\left(\begin{array}{l}
U_{u, x} \\
U_{u, y}
\end{array}\right) \leq \frac{1}{2} \sum_{w \in \mathcal{W}} W_{w} \omega_{b, w}+\left(1-\alpha_{s, b}\right) M_{s} \\
\forall b \in \mathcal{B}, \quad \sum_{s \in \mathcal{S}} \alpha_{s, b} \leq N_{s} a_{b} \\
\forall b \in \mathcal{B}, \quad \sum_{w \in \mathcal{W}} \omega_{b, w}=1 \\
\forall b \in \mathcal{B}, \quad \sum_{r \in \mathcal{R}} \rho_{b, r}=1
\end{gathered}
$$

$$
\forall b, b^{\prime} \in \mathcal{B} \text { such that } b^{\prime}>b, \quad \beta_{b, b^{\prime}}+\sum_{u \in \mathcal{U}} \gamma_{b, b^{\prime}, u} \geq 1
$$

$\forall b, b^{\prime} \in \mathcal{B}$ such that $b^{\prime}>b, \forall u \in \mathcal{U}$,

$\left(\begin{array}{l}x_{b^{\prime}}-x_{b} \\ y_{b^{\prime}}-y_{b}\end{array}\right)^{T}\left(\begin{array}{l}U_{u, x} \\ U_{u, y}\end{array}\right) \geq \frac{\kappa}{2} \sum_{w \in \mathcal{W}} W_{w}\left(\omega_{b, w}+\omega_{b^{\prime}, w}\right)-N\left(1-\gamma_{b, b^{\prime}, u}\right)$

(9)

$$
\begin{gathered}
\forall b, b^{\prime} \in \mathcal{B} \text { such that } b^{\prime}>b, \forall r \in \mathcal{R}, \\
\beta_{b, b^{\prime}}+\rho_{b, r}+\rho_{b^{\prime}, r} \leq 2+\left(1-a_{b}\right)+\left(1-a_{b^{\prime}}\right) \\
\forall b \in \mathcal{B}, \quad \sum_{s \in \mathcal{S}} T_{s} \alpha_{s, b} \leq \sum_{w \in \mathcal{W}} \Gamma_{w} \omega_{b, w}
\end{gathered}
$$

Variables: $\alpha_{s, b}, \omega_{b, w}, \rho_{b, r}, a_{b}, \beta_{b, b^{\prime}}, \gamma_{b, b^{\prime}, u} \in\{0,1\}$ and $x_{b}, y_{b} \in \mathbb{R}$

Fig. 7. Mixed Integer Linear Programming model devised for the beam layout optimization problem with linear Euclidean norm approximation

In Fig. 7, equations (2),(3),(4),(5) represent system level decisions and constraints: maximization of the covered traffic, a station is served at most by one beam, a station can be served by a beam only if the beam covers the station geometrically, and a non-active beam cannot serve any station. (6),(7),(8),(9),(10) are antenna considerations: each beam is assigned exactly one reflector and one beamwidth, and two active beams assigned to the same reflector must be spatially separated according the equation (1). Finally, equation (11) is the maximum load per beam defined per beamwidth to ensure the feasibility of the following repeater design phases. Even if each equation can be categorized, they well capture the interconnections of all the subsystems involved. For instance, the beamwidths intervene at all levels: the geometrical covering of the stations, the spatial separation antenna constraint, and the maximum load per beam repeater constraint. 


\section{FIRST EXPERIMENTAL RESULTS}

Experiments were conducted on the linear model of Fig. 7 to assess its performances. We generated a series of instances characterized by a growing number of user stations in input (progressive expansion of the service zone). In the results of Fig. 8, 168 instances were tested, with a total number of stations per instance growing from 190 to 858 . There were 4 available satellite reflectors, with a separation coefficient $\kappa=\sqrt{3}$. The traffic request of each station has been generated randomly and the upper-bounds of load per beam adjusted accordingly. Five beamwidths were defined: $\mathcal{W}=$ $\left\{0.35^{\circ}, 0.40^{\circ}, 0.45^{\circ}, 0.50^{\circ}, 0.55^{\circ}\right\}$. The number of discretized directions has been set to $n_{\text {directions }}=12$. The maximum number of beams has been adjusted three times according to the size of the instance (which is visible with the steps in figure (a) of Fig. 8). For all the runs, the MILP solver Gurobi has been used and we set a timeout to 600 seconds: the relative gap between the best solution and the best bound (best theoretical objective) is shown in figure (b) of Fig. 8. We observe

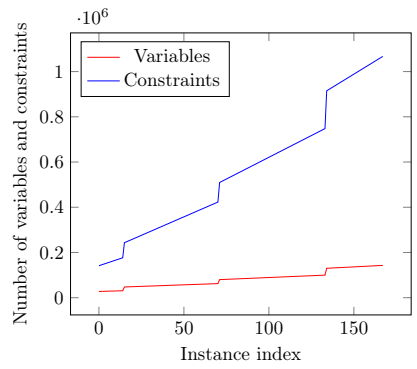

(a)

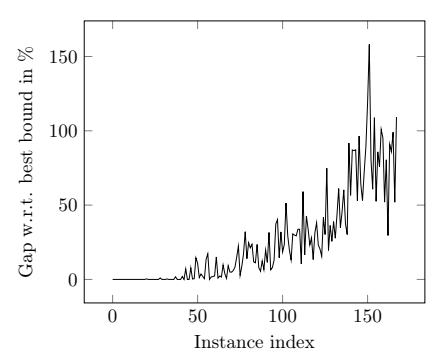

(b)
Fig. 8. (a) Growing numbers of variables and constraints (b) Gap with respect to the best bound after 600 seconds

that the model behaves extremely well on small instances with several instances being solved optimally (gap $=0 \%$ ) in 600 seconds. On larger instances, the results are more mitigated since there are cases were the solution reached is relatively far from the estimated best possible solution. Yet, the good results on small instances have motivated a comparison with the greedy heuristic solution of [3]. This comparison is natural since it is the only other known algorithm that considers the SFPB antenna separation constraints and that handles the beam centers actively. Fig. 9 summarizes their respective characteristics. An important remark is that, while our mixed-integer linear programming is exponentially impacted by the size of the instances (see subfigure (b) of Fig. 8), the complexity for the greedy heuristic grows linearly with respect to this input data size and allows to solve bigger instances. On the other hand, on small instances, our algorithm reaches and proves the optimality of the solution reached where the greedy algorithm only provides good but suboptimal solutions. This result should encourage the research on mixed-integer linear programming inspired techniques for the beam layout optimization.

\section{CONCLUSION AND WAY FORWARD}

The article allowed to highlight the complexity of designing multibeam satellite systems. A decomposition of the system design is therefore necessary and we proposed a detailed process for the space segment. The stress was put on the need to have explicit links between the different design steps, otherwise the solution produced might be infeasible or suboptimal in the end. To do so, we proposed to rely on mixed-integer linear programming to assist the design and the example analyzed here was the beam layout optimizaiton, for which a novel mathematical formulation has been presented and compared to an existing algorithmic solution. Excellent results were reached on part of the instances tested, but there is still work to do to overcome the numerical difficulties observed on the biggest

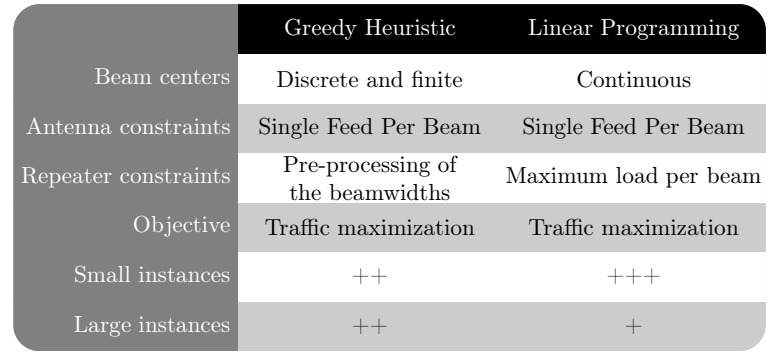

Fig. 9. Comparison of the greedy heuristic of [3] and of the mixed-integer linear programming approach for non-uniform beam layouts

instances. Therefore, the natural way forward is further research to enhance the current mixed-integer linear programming algorithm. The goal is either to solve higher instances more rapidly or to take advantage of that computational gain to solve in an even more integrated manner the different space segment design steps: the beam layout, the antenna, and the repeater design. To do so, there are promising advanced techniques worth investigating in operations research: Benders' decompositions, branch-and-price formulations and metaheuristics for instance.

\section{REFERENCES}

[1] P. Angeletti and J. Lizarraga. Non-regular multibeam coverage payloads for non-uniform traffic demand. $2^{\text {nd }}$ ESA Workshop on Advanced Flexible Telecom Payloads.

[2] M. Bousquet and G. Maral. Satellite Communications Systems : Systems, Techniques and Technology. $5^{\text {th }}$ edition, December 2009.

[3] J. Camino, C. Artigues, L. Houssin, and S. Mourgues. A greedy approach combined with graph coloring for non-uniform beam layouts under antenna constraints in multibeam satellite systems. Advanced Satellite Multimedia Systems Conference and the 13th Signal Processing for Space Communications Workshop (ASMS/SPSC), 2014.

[4] H. Chang. A simple proof of thue's theorem on circle packing. 2010.

[5] D. Christopoulos, S. Sharma, S. Chatzinotas, J. Krause, and B. Ottersten. Coordinated multibeam satellite co-location : The dual satellite paradigm. arXiv preprint arXiv:1503.06981, 2015.

[6] K. Kiatmanaroj, C. Artigues, and L. Houssin. On scheduling models for the frequency interval assignment problem with cumulative interferences. August 2013.

[7] K. Kiatmanaroj, C. Artigues, L. Houssin, and F. Messine. Frequency assignment in a SDMA satellite communication system with beam decentring feature. Computational Optimization and Applications, 56:439-455, 2013.

[8] A. Kyrgiazos, B. Evans, and P. Thompson. Irregular beam sizes and non-uniform bandwidth allocation in hts multibeam satellite systems. $31^{\text {st }}$ AIAA International Communications Satellite Systems Conference (ICSSC).

[9] U. Park, H. Kim, D. Oh, and B. Ku. A dynamic bandwidth allocation scheme for a multi-spot-beam satellite system. ETRI Journal, 2012.

[10] S. Poulenard, M. Ruellan, B. Roy, J. Ridi, F. Parol, and A. Rissons. High altitude clouds impacts on the design of optical feeder link and optical ground station network for future broadband satellite services. Free-Space Laser Communication and Atmospheric Propagation XXVI, 2014.

[11] C. Touati, E. Altman, J. Galtier, B. Fabre, and I. Buret. Radio planning in multibeam geostationary satellite networks. AIAA International Communication Satellite Systems Conference and Exhibit (ICSSC), 2003.

[12] T. Touya and D. Auroux. Control and topological optimization of a large multibeam array antenna. Fifth IASTED International Conference on Antennas, Radar and Wave Propagation, 2008.

[13] O. Vidal, G. Verelst, J. Lacan, E. Alberty, J. Radzik, and M. Bousquet. Next generation high throughput satellite system. IEEE First AESS European Conference on Satellite Telecommunications (ESTEL).

[14] H. Wang, A. Liu, X. Pan, and J. Li. Optimization of power allocation for a multibeam satellite communication system with interbeam interference. Journal of Applied Mathematics, 14, 2014. 